\title{
In-Line Inspection of Hot-Rolled Steel Billets by Heat Flux Thermography
}

\author{
by S. Koch*, J. Schroeder*
}

*Institut Dr. Foerster GmbH \& Co.KG; 72766 Reutlingen, Germany

\begin{abstract}
In this work heat flux thermography algorithms are proposed to suppress false indications that appear when testing steel billets with inductive thermography. Not only temperature amplitudes are used for evaluation but also the time dependancy of the temperature profile, different types of cooling rates and signal shape parameters. These new algorithms were tested successfully in a rolling mill. Hands-on experience will be presented as well as examples of noise signals that can be effectually suppressed.
\end{abstract}

\section{Introduction}

Since more than 30 years, inductive thermography is used for in-line inspection of hot-rolled steel billets [1]. In the past the image acquisition was done by scanning the billet surface with a single sensor element and a rotating mirror. Nowadays infrared cameras based on focal plane arrays (FPA) are state-of-the-art. Both types of detectors are actually used to discover local hot spots in the temperature profile of the billet surface which can be correlated with material flaws. For the first time, the method of heat flux thermography is introduced in the inspection of hot-rolled steel products. Each area of the billet surface is captured many times. For this reason not only hot spots can be detected. Also the time behaviour of the hot spots can be analysed. Examples will show that not all hot spots automatically correlate to material flaws but are false indications. These can be caused by inadequate surface properties or inappropriate testing conditions. Heat flux thermography has the ability to suppress false indications to minimise needless reworking.

\section{Inductive Thermography}

\subsection{Background}

Along the production of semifinished steel products the material is often subject to extreme loads. These are thermal strains in case of casting and hardening, but also mechanical strains along rolling. On the other side the requirements on the material quality are increasing continously. Therefore, a variety of testing methods is available. Thermography has the advantage that it is contactless and nearly independent of the material shape. It can be used for an automatic evaluation although the testing speeds for metals are rather high.

\subsection{Testing Setup}

For testing the billets they undergo the following procedure (Fig. 1). In a first step the surface is sprayed with water to raise the emissivity coefficient $\varepsilon$ of the surface and make it homogenous. In a second step the billet is heated up by an induction coil that is driven by high frequency currents. According to the skin effect cracks or other structural flaws on the material surface act as barriers for the generated eddy currents. 


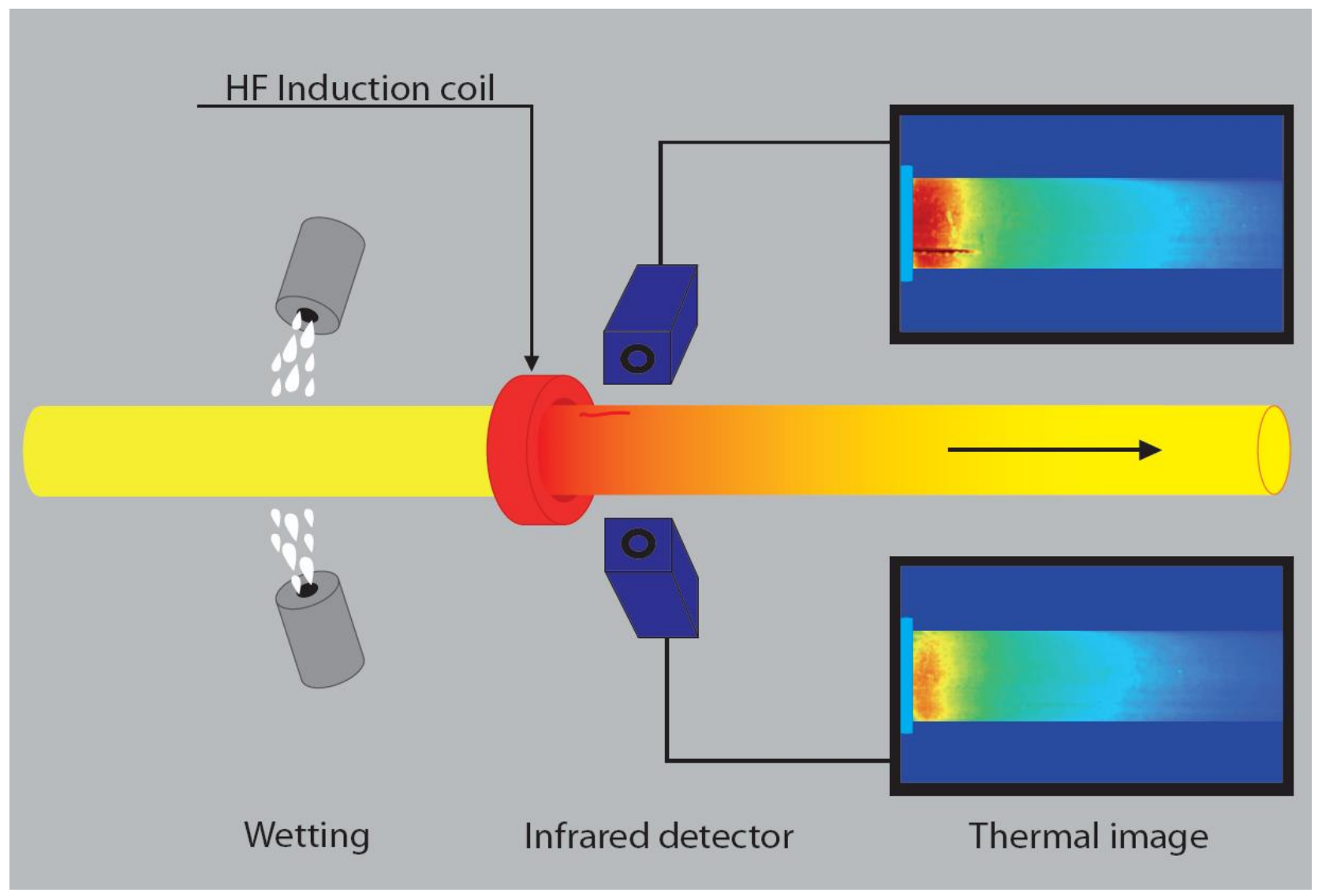

Fig.1. Principle of testing setup

At these bottlenecks the local current density and therefore the corresponding heat losses are higher than on the flawless parts of the surface. In a third step the resulting temperature peaks can be captured by means of high speed infrared cameras whereas the temperature signals are evaluated by computers with specialised algorithms. According to the computer results the flaws are marked and the corresponding billets are sorted out e.g. for grinding.

\section{Heat Flux Thermography}

In daily practice false indications induced by emissivity variations and other inappropriate testing conditions are still a handicap when using thermography in industry because they lead to needless reworking and wear of grinding equipment. The reason for these false indications is that profiles of cracks and other ,events' like e.g. reflections appear similar (Fig. 2).
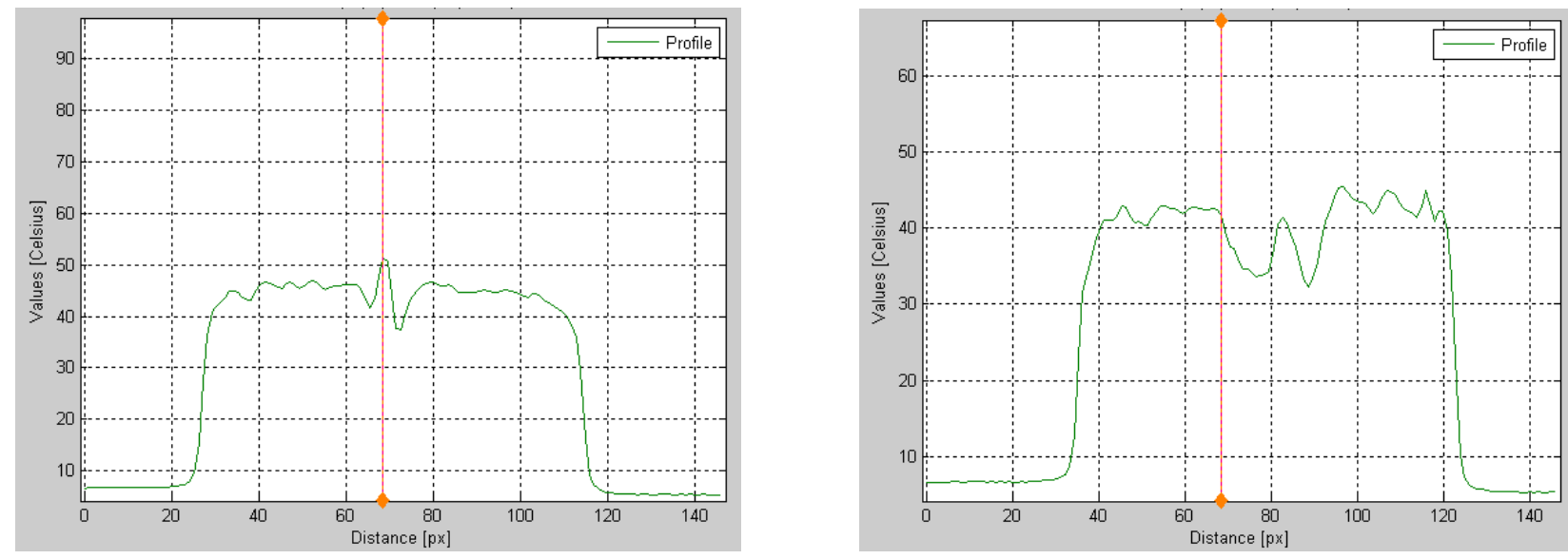

Fig.2. Crack signal of defect with $0.3 \mathrm{~mm}$ depth (left), noise signal induced by a reflection (right) 
Heat flux thermography provides an approach to overcome this problem. Each position on the billet surface is captured up to 30 times when going through the field of view of the infrared camera. The flow of heat then can be followed and evaluated (Fig. 3).
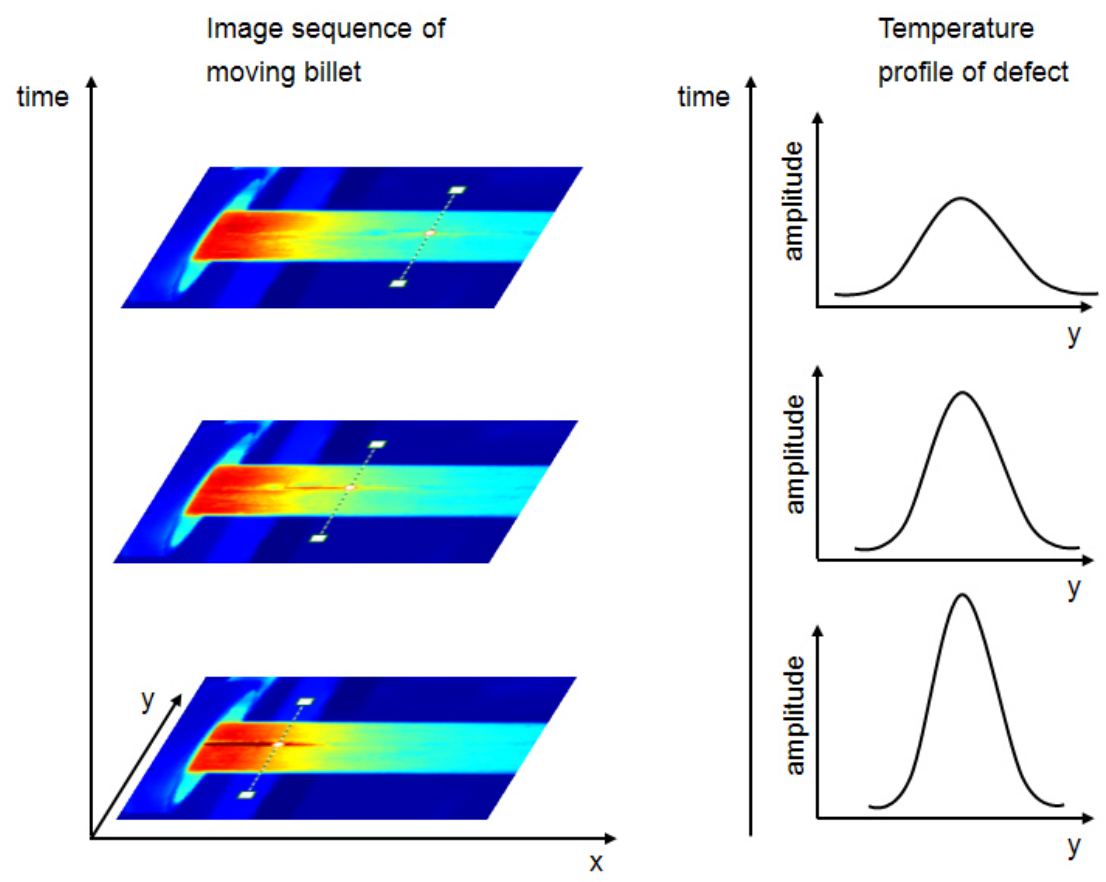

Fig.3. Time evolution of temperature signal

New criterions were developed that decide if the time behaviour of an 'event' corresponds to a real crack or another physical property of the billet surface. These criterions are the cooling and the broadening rates of the temperature signal, the shape factor of the signal and the characteristics of the time behaviour itself.

\section{Case Study}

In the following, three typical scenarios will be presented that show how the parameters signal amplitude and heat concentration in the 'defect' area behave.

\subsection{Crack signal}
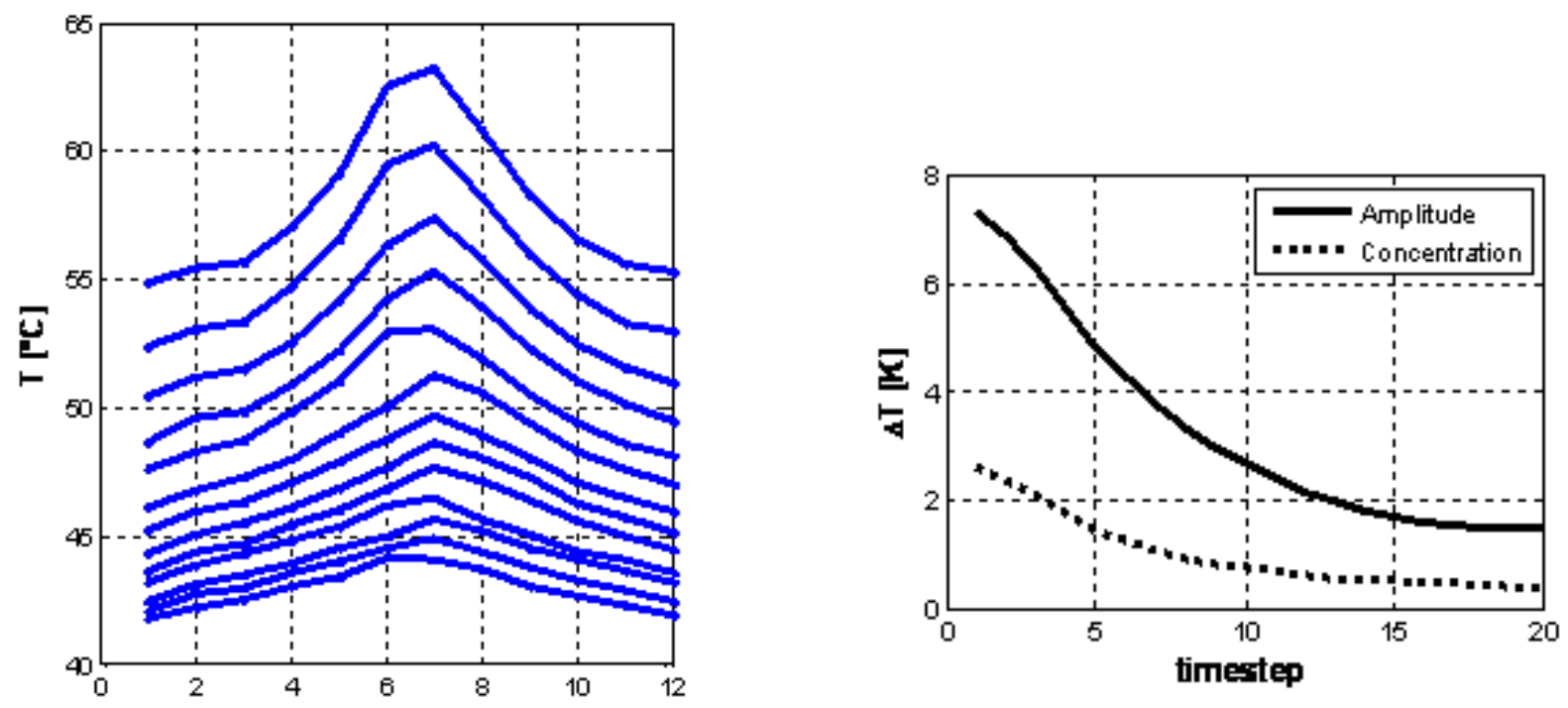

Fig.4. Defect profile at different times (left), evolution of defect amplitude and heat concentration, i.e. width of the defect signature (right) 
A typical crack behaves like in Fig. 4. The amplitude decreases with time (here 13 time steps are shown). Additionally the signal is getting broader and the heat concentration around the defect is getting smaller. Now one can define thresholds for minimum amplitude, minimum cooling rate of the amplitude, minimum broadening rate of the signal and additional shape parameters to discriminate against false indications that are described in 4.2 and 4.3.

\subsection{Noise (Case 1)}
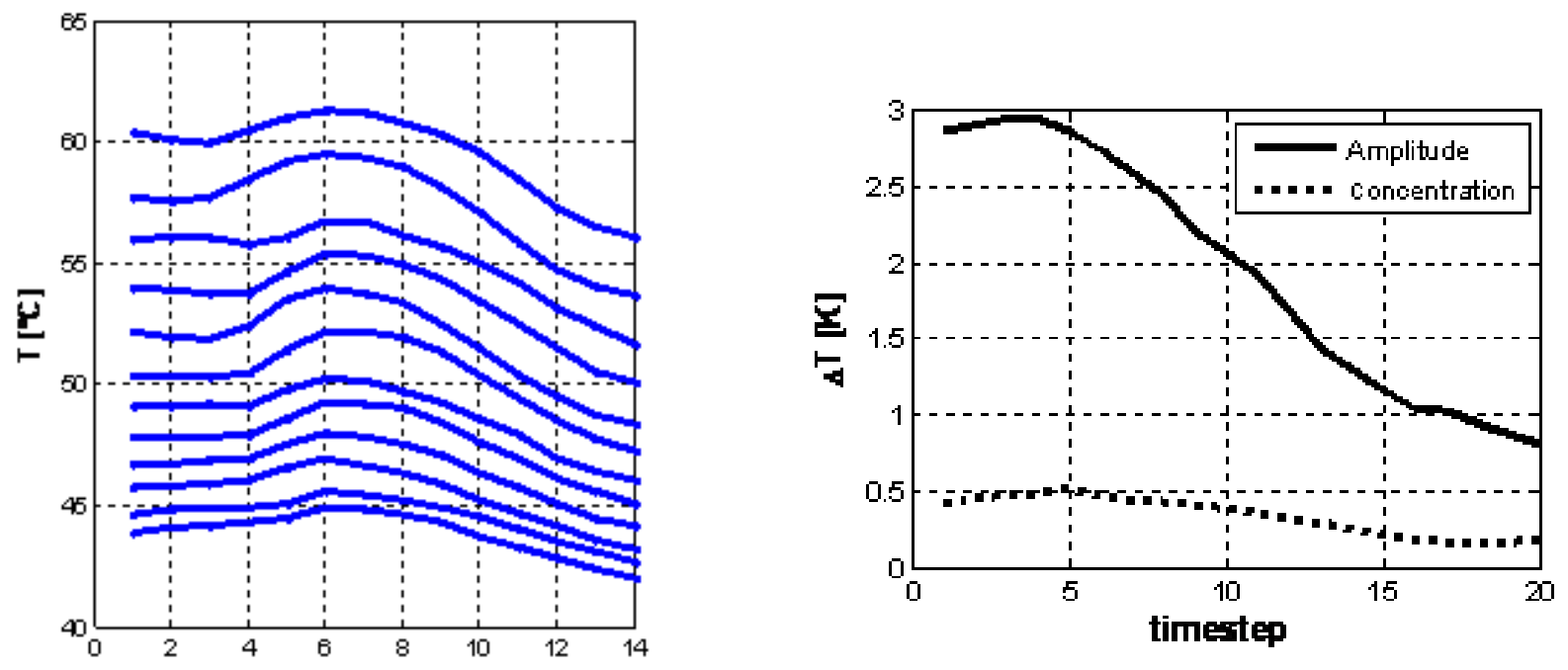

Fig.5. Broad amplitude (left), therefore small heat concentration (right)

The signals from Fig. 5 originate from rolling marks. The amplitude of the noise signal behaves like a crack, i.e. it decreases with time. The amplitude threshold will not help to discriminate against this false indication. The same holds for the cooling rate. The broadening of the signal is slow compared with 4.1. If one sets the threshold in a way that a crack should behave like in 4.1, this criterion would reject this kind of 'event'.

\subsection{Noise (Case 2)}
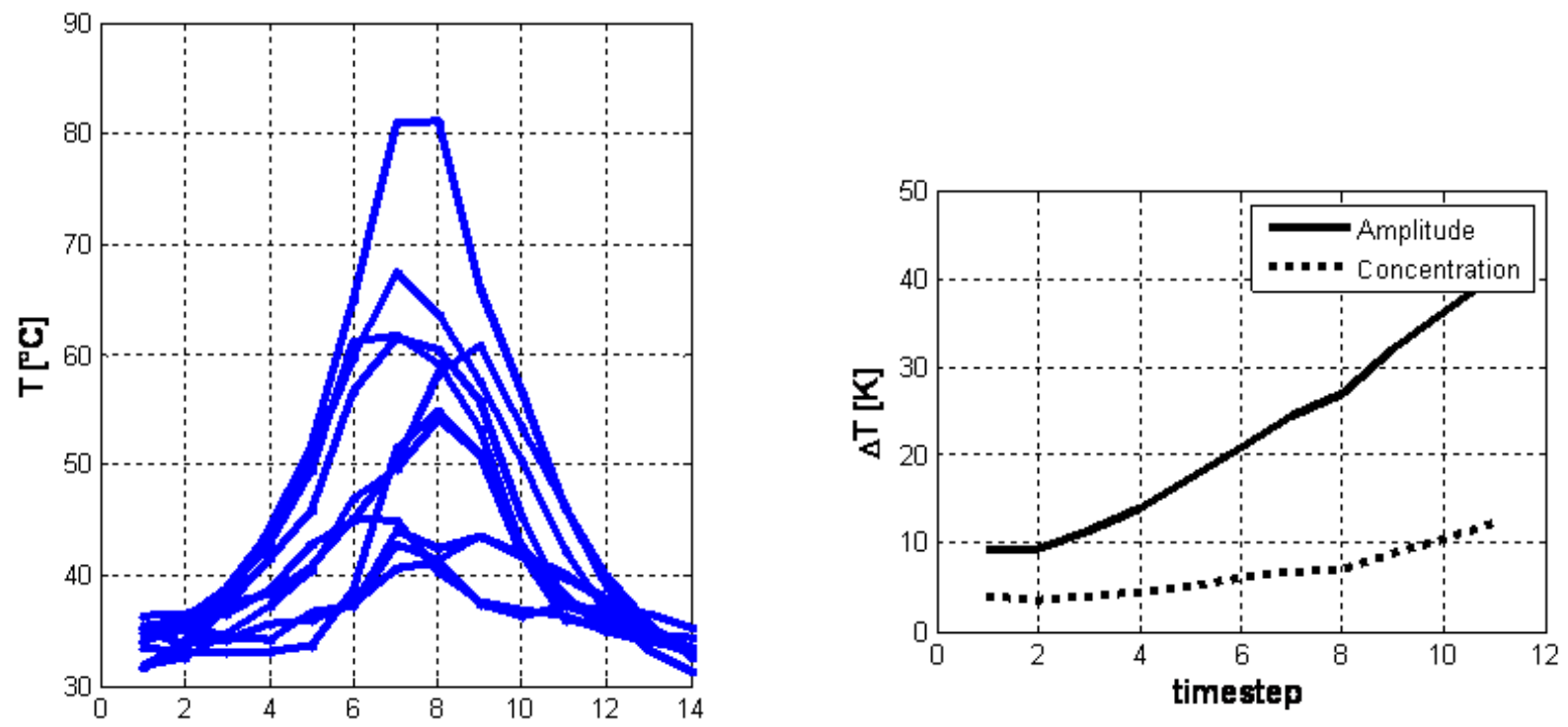

Fig.6. Increase of signal amplitude (left, right), also increase of heat concentration (right)

The signals from Fig. 6 result from reflections of an overhead radiation heating on the steel billet. The amplitude is growing instead of decreasing. Also the signal is getting sharper. Here both, the cooling rate and the broadening rate will reject the 'event'. 


\section{Conclusions}

Inductive thermography is already a helpful technology for detecting smallest cracks and other kind of material flaws in steel billets. It is a contactless and imaging method that can be easily automated.

In this presentation it was shown that taking the time behaviour of the temperature signals into account improves the reliability and stability of the testing procedure by minimising the amount of false indications. Heatflux thermography assists to save precious time in terms of reworking and minimises the consumption of grinding tools.

\section{REFERENCES}

[1] M. Storset, 'THERM-O-MATIC Billet inspection system', SCANCONDITIONING, $1^{\text {st }}$ International Conference on Surface Conditioning and Detection of Surface Defects, Proceedings, pp 2:1-2:24, June 1984 